\title{
Overexpression of OsFTL10 induces early flowering and improves drought tolerance in Oryza sative $L$.
}

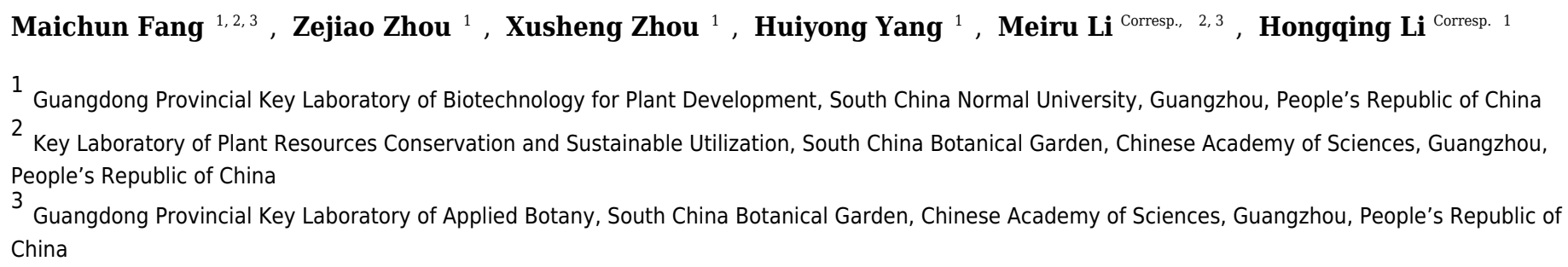

Flowering time control is critically important for the reproductive accomplishment of higher plants as floral transition can be affected by both environmental and endogenous signals. Flowering Locus T-like ( $F T L)$ genes are major genetic determinants of flowering in plants. In rice, 13 OsFTL genes have been annotated in the genome and amongst them, $\mathrm{Hd} 3 \mathrm{a}$ (OSFTL2) and RFT1 (OSFTL3) have been studied extensively and their functions are confirmed as central florigens that control rice flowering under short day and long day environment, respectively. In this report, a rice OsFTL gene, OsFTL10, was characterized, and its function on flowering and abiotic stress was investigated. The expression level of OsFTL10 was high in young seedlings and shown to be induced by GA3 and drought stress. Overexpression of OSFTL10 resulted in earlier flowering in rice plants by up to 2 weeks, through up-regulation of the downstream gene OSMADS15. OsFTL10 also regulated Ehd1 and OsMADS51 through a feedback mechanism. The OsFTL10 protein was also detected in both nucleus and cytoplasm. Furthermore, yeast two hybrid $(\mathrm{Y} 2 \mathrm{H})$ and bimolecular fluorescence complementation (BiFC) results show that OsFTL10 could interact with multiple 14-3-3s, suggesting that OsFTL10 might function in a similar way to $\mathrm{Hd} 3 \mathrm{a}$ in promoting rice flowering by forming a FAC complex with 14-3-3, and OsFD1. Further experiments reveal that constitutive expression of OSFTL10 improved the drought tolerance of transgenic plants by stimulating the expression of drought responsive genes. These results suggest that rice $F T L$ genes might function in flowering promotion and responses to environmental signals. 


\section{Title}

2

\section{Overexpression of $O$ sFTL10 induces early flowering and improves}

4 drought tolerance in Oryza sative L.

5

6 Maichun Fang, ${ }^{\mathrm{a}, \mathrm{b}, \#}$, Zejiao Zhou ${ }^{\mathrm{a} \#}$, Xusheng Zhou ${ }^{\mathrm{a}}$, Huiyong Yang ${ }^{\mathrm{a}}$, Meiru Li ${ }^{\mathrm{b}, \mathrm{c}^{*}}$, Hongqing

$7 \quad \mathbf{L i}^{\mathrm{a}^{*}}$

$8{ }^{a}$ Guangdong Provincial Key Laboratory of Biotechnology for Plant Development, South China

9 Normal University, Guangzhou,510631, People's Republic of China

10 b Key Laboratory of Plant Resources Conservation and Sustainable Utilization, South China

11 Botanical Garden, Chinese Academy of Sciences, Guangzhou 510650, People's Republic of

12 China

$13^{\mathrm{c}}$ Guangdong Provincial Key Laboratory of Applied Botany, South China Botanical Garden,

14 Chinese Academy of Sciences, Guangzhou 510650, People’s Republic of China

15

16

17

18

19

20

21

22

23

24

25

26

27

28

\#These authors have contributed equally to this work.

${ }^{*}$ Correspondence

Hongqing Li

Guangdong Provincial Key Laboratory of Biotechnology for Plant Development, South China Normal University, Guangzhou 510631, People’s Republic of China

Email address: hqli@scnu.edu.cn 


\section{ABSTRACT}

30

31

32

Flowering time control is critically important for the reproductive accomplishment of higher plants as floral transition can be affected by both environmental and endogenous signals. Flowering Locus T-like $(F T L)$ genes are major genetic determinants of flowering in plants. In rice, 13 OsFTL genes have been annotated in the genome and amongst them, Hd3a (OsFTL2) and RFT1 (OsFTL3) have been studied extensively and their functions are confirmed as central florigens that control rice flowering under short day and long day environment, respectively. In this report, a rice $O S F T L$ gene, OSFTL10, was characterized, and its function on flowering and abiotic stress was investigated. The expression level of OSFTL10 was high in young seedlings and shown to be induced by GA3 and drought stress. Overexpression of OsFTL10 resulted in earlier flowering in rice plants by up to 2 weeks, through up-regulation of the downstream gene OsMADS15. OsFTL10 also regulated Ehd1 and OsMADS51 through a feedback mechanism. The OsFTL10 protein was also detected in both nucleus and cytoplasm. Furthermore, yeast two hybrid (Y2H) and bimolecular fluorescence complementation (BiFC) results show that OsFTL10 could interact with multiple 14-3-3s, suggesting that OsFTL10 might function in a similar way to Hd3a in promoting rice flowering by forming a FAC complex with 14-3-3, and OsFD1. Further experiments reveal that constitutive expression of OsFTL10 improved the drought tolerance of transgenic plants by stimulating the expression of drought responsive genes. These results suggest that rice $F T L$ genes might function in flowering promotion and responses to environmental signals. 


\section{INTRODUCTION}

54

55

56

57

58

59

60

61

62

63

64

65

66

67

68

69

70

71

72

73

74

75

76

Flowering is one of the most important physiological processes that changes the vegetative and reproductive growth of plants. Floral transition can be induced by both environmental factors and endogenous genetic networks. Upon flowering induction, multiple signals converge in floral pathway integrators which subsequently activate downstream genes for growth phase transition and the development of flower organs (Wagner 2017). Recently, advances in the molecular genetics of Arabidopsis and rice, have revealed the molecular nature of florigen to be a globular protein, named FLOWERING LOCUS T (FT), which is produced in the mature leaf and transported to shoot meristem to induce flowering. This process satisfies the major prerequisites of florigen as a systemic floral signal (Corbesier et al. 2007; Tamaki et al. 2007).

$H d 3 a$, the Arabidopsis FT homolog protein in rice, belongs to the phosphatidylethanolamine binding protein (PEBP) family in animals (Kardailsky et al. 1999). Both $H d 3 a$ and FT are expressed in mature leaves when flowering-promotive day lengths are given, long days (LD) for Arabidopsis and short days (SD) for rice (Corbesier et al. 2007; Tamaki et al. 2007). Both proteins migrate from leaves to the apical meristem (SAM), where they bind to transcription factors of the bZIP family, including FD in Arabidopsis and OsFD1 in rice. The rice Hd3a and FD1 have been shown to form a florigen activation complex (FAC) with 14-3-3 (GF14 in rice) to promote floral initiation (Tamaki et al. 2007; Taoka et al. 2011).

Genome annotation results have revealed thirteen FT homologs (OsFT-LIKE genes) in the rice genome (Chardon and Damerval 2005). Amongst the thirteen FTLs, RFT1/FT-L3 showed the highest homology with $\mathrm{Hd} 3 \mathrm{a}$, and was recently found to be important for rice flowering under LD conditions. FTL/FT-L1 is another member with high homology to Hd3a. Transgenic rice plants overexpressing RFT1 or FTL exhibited an early flowering phenotype, similar to $H d 3 a$ - 
77 overexpressing plants (Izawa et al. 2002; Kojima et al. 2002). Knockdown of Hd3a or RFT1 in the 78 japonica rice cultivar Norin 8, delays rice flowering only under SDs or LDs, respectively (Komiya 79 et al. 2008, 2009). In contrast, flowering in rice is almost completely blocked even under favorable 80 SD conditions by knockdown of both $H d 3 a$ and RFT1, suggesting that these two genes have 81 overlapping functions and are necessary for flowering (Komiya et al. 2008). Until now, studies in 82 rice have revealed that the photoperiod is a major environmental factor influencing rice flowering 83 time. Furthermore, photoperiodic flowering in rice is considered as two distinct pathways: one 84 including OsGI (an orthologue of Gigantea ),Hd1(Heading date 1) and Hd3a. These proteinsform 85 a pathway of OsGI-Hd1-Hd3a, which is similar to that of Arabidopsis under SD conditions (Turck et al. 2008, Deng et al.2017). Another one including Ghd7 (Grain Number, Plant Height and 87 Heading Date 7 ), Ehd1 (Early heading date 1) and Hd3a/RFT1.These proteins form a pathway of 88 Ghd7-Ehd1-Hd3a/RFT1 in rice under LD conditions. The major genes downstream of Hd3a and RFT1 are OsMADS14, OsMADS15, OsMADS18 and OsMADS34. Simultaneous knockdown of the above four genes completely blocked flowering in rice (Kobayashi et al. 2012; Tsuji 2017).

Floral transition can be induced by a variety of external factors, including various environmental conditions and hormone treatments, such as the well-studied photoperiod pathway in rice and Arabidopsis, as well as the vernalization and $\mathrm{GA}_{3}$ (Gibberellic Acid) pathway in Arabidopsis (Kazan and Lyons 2016). Drought stress influences plant growth and crop yield in many areas of 95 the world, and has been shown to significantly influence flowering 96 time. During drought stress, plants may adopt the strategy of 'drought escape' by speeding up or 97 delay of flowering (Bernal et al. 2011; Franks 2011), or the strategy of 'drought avoidance' or 98 'drought tolerance' by reducing water loss to prevent dehydration (Kooyers 2015). In Arabidopsis, 99 drought stress affects flowering time through transcriptional regulation of FT and 
100 TWINSISTER OF FT (TSF). Under long day conditions, induction of the expression of 101 FT and TSF by drought stress depends on GI and abscisic acid (ABA), whilst under 102 short days, floral repressors are induced by ABA and drought, and 103 the transcription of TSF and FT is inhibited (Riboni et al. 2013). The Grain Number, Plant Height 104 and Heading Date 7 (Ghd7), which delays heading and increases yield in rice under long days 105 (Xue et al. 2008), also affects drought tolerance in rice (Weng et al. 2014, Kazan and Lyons 2016). 106 In rice, the function of FTL genes on the induction of flowering has been well-characterized based 107 on the studies of $H d 3 a$ and RFT1. The precise involvement of florigens in environmental response 108 is largely unknown. When investigating the response of rice FTLs to hormones and abiotic stresses, 109 we found that only OsFTL10 expression was stimulated by mannitol and ABA. In this report, we 110 studied the expression of OSFTL10 and found that it expressed at a higher level in young leaf, and 111 also its expression can be induced by drought stress. Overexpression of OSFTL10 can induce early 112 flowering in rice through a similar mechanism as that of $\mathrm{Hd} 3 \mathrm{a}$. We also found that overexpression 113 of OsFTL10 can enhance the drought tolerance of plants.

116

117

118

119

120

121

122

123

\section{MATERIALS \& METHODS}

Vector construction and rice transformation

To obtain the OsFTL10 overexpression construct, a fragment containing the complete OsFTL10 cDNA (Os05g0518000) was amplified with the primer set Fw-OsFTL10-EcoRI/Re-OsFTL10EcoRI (Supplemental Table S1), and cloned into the EcoRI site in binary expression vector pCAMBIA1390. To make the RNAi construct, the 
124 cDNA fragment was amplified with the primer set Fw-OsFTL10-RNAi/ Re-OsFTL10-RNAi, and 125 cloned into the pTCK303 vector (Wang et al. 2004).

126 To examine the promoter activity in rice, the OsFTL10 promoter (pFTL10), spanning about 2000 127 bp upstream of the ATG start codon of OsFTL10 was amplified using PCR. The CaMV35S 128 promoter for $\beta$-glucuronidase (GUS) expression in pCAMBIA1301 was replaced with 129 pFTL10. For subcellular localization studies of OsFTL10, its cDNA fragment was amplified by 130 PCR and fused with EGFP in the binary vector 1390. The binary vector for expression of $H d 3 a$ 131 was from a previous work ( $\mathrm{Li}$ et al, 2011). The above constructs were introduced into 132 Agrobacterium tumefaciens strain EHA105 by electroporation (Li et al. 2011). Agrobacterium133 mediated transformation of rice (Oryza sativa L. ssp. japonica., Zhonghua 11) was performed 134 as described (Li et al. 2011).

135

136 Plant materials and growth conditions

137

138 Mature seeds of Zhonghua 11 (Oryza sativa L. ssp. japonica.) were collected from WT and 139 homozygous T2 transgenic plants. Seeds were germinated for 2 days at $28^{\circ} \mathrm{C}$ in the dark, after 140 which they were planted in soil and grown under greenhouse conditions to maturity (12-h light at $14130{ }^{\circ} \mathrm{C} / 12-\mathrm{h}$ dark at $22^{\circ} \mathrm{C}$ ). Mature leaves from 30 day old plants were collected at $10 \mathrm{O}^{\prime}$ clock in 142 the morning, and RNAs were extracted for qRT-PCR analysis of the expression of flowering 143 related genes. The major agronomic traits including heading date, plant height, grain weight and 144 number of flowers per panicle of the homozygous plants were investigated.

145

146 FTL10 sub-cellular localization and BiFC analysis 
148 The subcellular localization of FTL10 and GF14c, as well as BiFC analysis characterizing the 149 interaction of FTL10 and GF14c, were carried out according to Lin, et al. 2017. For subcellular 150 localization studies of FTL10 and GF14c, the coding regions of both FTL10 and GF14c were 151 amplified with primers (Supplemental Table S1) and fused with EGFP, respectively. For BiFC 152 analysis, GF14c and OsFTL10 were amplified by PCR and restriction-cloned (BamHI and/or 153 EcoRI) into the p35S::cYFP and p35S::nYFP vectors, respectively. Negative controls constructs 154 were made by replacing GF14c or OsFTL10 with GUS (Taoka et al. 2011). The above vectors were transformed into rice protoplasts for live cell imaging. Images were captured using a LSM 156710 confocal microscope (Zeiss, Jena, Germany) (Lin, et al. 2017).

Yeast two-hybrid assays

159

Yeast two-hybrid (Y2H) assays were performed using the Matchmaker ${ }^{\mathrm{TM}}$ Gold Yeast Two-Hybrid 161 Systems (Clontech) according to Lin et al. (2017). The open reading frames of OsFTL10 and 143-3s were inserted into pGBKT7 and pGADT7 vectors with proper restriction enzyme sites indicated in the primers to create bait and prey, respectively.

GUS staining of transgenic rice plants

167 Homozygous transgenic lines carrying the pFTL10::GUS were randomly chosen for the study.

168 Seeds were briefly sterilized and germinated, then planted in soil and grown under greenhouse 
169 conditions. Immature and mature leaf, roots and other tissues were collected from the plants

170 grown at different stages, GUS staining of the plant materials was performed according to

171 procedures described by Jefferson (1987).

172

173 Growth regulators and abiotic stress treatments

174

175 WT seedlings germinated for 2 weeks on half strength MS medium were collected and incubated 176 in $\mathrm{ddH}_{2} \mathrm{O}$ containing either of the following components: $5 \mathrm{mmol} / \mathrm{L}$ IAA, $1 \mathrm{mmol} / \mathrm{L}$ 6-BA, 0.1 $177 \mathrm{mmol} / \mathrm{L} \mathrm{MeJA}, 10 \mathrm{mmol} / \mathrm{L} \mathrm{GA}_{3}, 100 \mu \mathrm{mol} / \mathrm{L} \mathrm{ABA}, 1 \mathrm{mmol} / \mathrm{L} \mathrm{SA}, 200 \mathrm{mmol} / \mathrm{L}$ Mannitol , 200

$178 \mathrm{mmol} / \mathrm{L} \mathrm{NaCl}$, respectively. Treatments with IAA and $\mathrm{BA}$ were performed for $0,0.5,1$ and 4 179 hours, respectively. Treatments with ABA, SA, MeJA and GA were performed for 0, 1, 4 and 12 180 hours, respectively. Treatments with mannitol and $\mathrm{NaCl}$ were performed for $0,6,14$ and 48 hours, 181 respectively. All the treatments were started from about 10 O'clock in the morning. After treatment 182 for the indicated times, total RNAs were extracted and reversely transcribed as the template for 183 qRT-PCR.

184

qRT-PCR for gene expression analysis

187 Total RNAs were extracted using a TRIzol kit according to the user's manual (Invitrogen). For 188 each sample, $1 \mu \mathrm{g}$ of total RNAs was reverse-transcribed as cDNA template using a kit (TaKaRa). 
189 qRT-PCR was carried out in a mixture of $20 \mu \mathrm{L}$ consisting of cDNA, gene specific primers 190 (Supplementary Table 1) and $10 \mu \mathrm{L} 2 \mathrm{X} \operatorname{mix}$ (TaKaRa), water was added to the mixture to a final 191 of 20 ul. qPCR was performed on an ABI 7900 real-time PCR machine according to the 192 manufacturer's instruction (Applied Biosystems). For each sample, three biological replicates 193 were performed and cDNA were amplified in triplicate by quantitative PCR. The relative 194 expression values were determined by using rice Ubiquitin gene as reference and the comparative 195 Ct method (2- $\Delta \Delta \mathrm{Ct})$.

196

197 Drought stress treatment

Drought stress treatment of WT and transgenic plants was performed according to Li et al. (2011).

200

201 202 203 204 205 206 207 208 209
Seeds of WT, overexpression transgenic lines ox2, ox5, and ox8 and RNAi lines i1,i2 and i5 (T3, homozygous seeds) were germinated, and in the same pot containing vermiculite were soaked with distilled water. Eight days later, water was withheld from the seedlings. After another 20 (for RNAi lines) or 25 days (for overexpression lines), the plants were re-watered for recovery. Three days later, plants with green, healthy leaves were regarded as viable, having survived. For study of drought related gene expression in transgenic plants, seedlings germinated for 2 weeks were incubated in a solution containing $200 \mathrm{mmol} / \mathrm{L}$ mannitol for 2, 4, 8, and 24 hours, respectively. Total RNA was extracted for qRT-PCR. 


\section{RESULTS}

211

212 Expression pattern of OsFTL10 in rice

213

214 OSFTL10 encodes a protein consisting of 174 amino acid residues. To investigate the temporal and 215 spatial gene expression pattern of OSFTL10 in rice, we quantified the OSFTL10 expression level 216 in different tissues including immature and mature leaves, stem, young panicle and floral organs 217 from the wild-type plants grown under SD conditions. OsFTL10 expression was detected in all the 218 above tissues. The highest expression level was observed in immature leaves with a lower level of 219 expression found in floral organs (Fig. 1A).

220 The expression pattern of OsFTL10 was also investigated by GUS staining assay in stable 221 transgenic plants. The promoter region of OSFTL10 was cloned and a construct was made by 222 placing the GUS reporter under the OSFTL10 promoter. The construct was transformed into rice 223 cell and stable transgenic plants were obtained. GUS expression was found in immature leaf, 224 mature leaf, flower, young panicle, pollen and shoot tip (Fig.1B, C, D, E, F, G, H). Strong GUS 225 staining was found in immature leaf tissue and very faint blue staining was found in the pollen 226 (Fig.1G).

227

228 Expression of OSFTL10 under growth regulators and abiotic stress treatments

230 To determine whether OsFTL10 expression is influenced by environmental factors, qRT-PCR was 231 performed to study the expression of OSFTL10 under different growth regulators and abiotic stress 232 treatments. Seedlings germinated for 2 weeks on half strength MS medium were collected and 
233 treated with different growth regulators and abiotic stresses for different time intervals, and total

234 RNAs were extracted for qRT-PCR. Amongst the treatments of different growth regulators, ABA

235 at $100 \mu \mathrm{M}$ stimulated the expression of OsFTL10 to nearly two-fold after $4 \mathrm{hrs}$ (Fig.2C). The

236 addition of $\mathrm{GA}_{3}$ at $10 \mathrm{mM}$ greatly increased the expression of OsFTL10 to more than ten-fold its

237 basal level (Fig.2F). Treatments with SA and MeJA also showed slightly increased expression of

238 OsFTL10 (Fig.2D, E), whilst treatment with IAA or BA reduced the expression level of OsFTL10

239 (Fig.2A, B). Abiotic stress treatment was performed using $200 \mathrm{mM}$ mannitol or $200 \mathrm{mM} \mathrm{NaCl}$.

240 Treatment with $200 \mathrm{mM}$ mannitol resulted in increased OsFTL10 expression to four-fold basal

241 levels at $6 \mathrm{hrs}$, and the expression dropped after $24 \mathrm{hrs}$ (Fig.2G). $200 \mathrm{mM} \mathrm{NaCl}$ slightly increased

242 the expression of OsFTL10 which dropped after $24 \mathrm{hrs}$ (Fig. 2H). In accordance with the above

243 results, some cis elements involved in drought stress and ABA response were found in OsFTL10

244 promoter (Supplemental File 1). As ABA and mannitol are related to drought stress, we further 245 studied the drought tolerance of OSFTL10 transgenic plants in addition to its function in flowering.

247 Overexpression of FTL10 induced earlier flowering in rice

248

249 As OsFTL10 is one of the homologs of $H d 3 a$, we expected that overexpressing this gene would 250 also promote rice flowering. We generated OSFTL10 overexpression transgenic plants and 251 examined the transgene expression by RT-PCR (Fig.3A). At the same time, we obtained RNAi 252 transgenic plants for OsFTL10, and in some lines OsFTL10 expression was greatly reduced (Fig. 253 3B). Representative pictures of the overexpressing line ox8 (Fig.3A) and the RNAi line (Fig.3B) 254 are shown. To investigate the agronomic traits of the transgenic plants, the homozygous T3 255 generation of the three independent overexpressing lines ox2, ox 5, ox 8 and RNAi lines i1, i2 ,i5 
256 were grown under short day conditions, and their phenotype data compared with WT plants (Fig.

257 3). The average heading date of the overexpressing lines was 53 days, which was about 2 weeks

258 less than of the WT plants (about 68 days). The RNAi lines did not show significant difference in

259 heading time compared with the WT (Fig. 3C). The plant height for the overexpressing lines was

260 on average $65 \mathrm{~cm}$ (Fig. 3D), which was about $19 \mathrm{~cm}$ shorter than that for the WT $(84 \mathrm{~cm})$. The

261 number of grains per panicle and grain weight were also reduced significantly in the

262 overexpressing lines, compared to that in WT (Fig. 3E, F). In the RNAi lines, the above traits were

263 not significantly changed (Fig. 3C, D, E, F). These results showed that overexpression of OsFTL10

264 could induce early flowering, which influenced the agronomic traits of the plants. The down-

265 regulation of endogenous OsFTL10 exerted little effect on plant growth. When compared with

$266 H d 3 a$ overexpression plants, we found that heading time of OsFTL10 overexpression plants were

267 about 3 weeks longer (Supplimental Table 1).

268 To elucidate the function of OSFTL10 on flowering induction, we first examined the subcellular

269 localization of the OsFTL10 and GF14c protein. Constructs were made to fuse the OsFTL10 or

270 GF14c with EGFP protein, under the CaMV35S promoter (CaMV35S::OsFTL10-EGFP). The

271 constructs were transformed into rice leaf mesophyll protoplasts, and the EGFP signal was

272 analyzed. The EGFP signal of OsFTL10 was observed in both the nucleus and cytoplasm, whilst

273 the EGFP signal of GF14c was observed mainly in the cytoplasm (Fig.4A). These results indicated

274 that FTL10 had a similar subcellular localization to Hd3a.

275 Hd3a was found to interact with 14-3-3, and form a complex with OsFD1 in the nucleus for 276 activation of the downstream flowering genes (Taoka et al. 2011). The interaction of OsFTL10 277 with GF14c in the rice protoplast was examined by BiFC. A strong signal was detected in the 
278 nucleus, which demonstrated the interaction of the two proteins. At the same time, the negative 279 controls showed no obvious signal (Fig.4B). The interactions of OsFT10 with multiple 14-3-3s 280 were also investigated by the yeast two-hybrid system. The results showed that OsFTL10 281 interacted with different 14-3-3 homologs in rice, including GF14a, GF14b, GF14c, GF14d, 282 GF14e (Fig.4C). These results suggested that OsFTL10 functioned similarly to Hd3a to bind with 283 14-3-3s, which was a key step for entering the nucleus in the formation of the FAC complex with 284 OsFD1 (Taoka et al. 2011).

285 Flowering in rice is mainly influenced by the photoperiodic pathway (Tsuji et al., 2011). OsGI (an 286 orthologue of Gigantea) is an integrator of photoperiod pathway in rice (Kim et al., 2008). Under 287 short day (SD) conditions, OsGI up-regulates the expression of $H d 1$ (a CONSTANS-like gene), 288 and $H d 1$ will induce the expression Ghd8 (grain yield, heading date and plant height 8 ). Ghd8 encodes the OsHAP3 subunit of a CCAAT-box binding protein and can activate the expression of 290 Hd3a (Yan et al., 2011). Ehdl (Early heading date 1) is a B type response regulator, which up291 regulates the expression of FT-like genes (Matsubara et al., 2011). OsGI can also up-regulate Ehdl 292 through activating MADS51 under SD conditions (Kim et al., 2008). To dissect the genetic 293 networks of OsFTL10 on flowering, we performed qRT-PCR analysis of the genes that played important roles in rice flowering. The cDNA template was obtained from RNA extracted from 295 mature leaves of WT, overexpressing lines ox 2 and ox8, and the RNAi lines i2 and i5 before heading. For genes upstream of $H d 3 a$ and RFT1, GI, Hd1, Ehd1 and MADS51 were selected. qRT297 PCR results showed that overexpression of FTL10 had no significant influence on the expression 298 of $G I$ and $H d 1$ in both ox 2 and ox 8, as their expression levels were similar to those in WT (Fig.5A, B) . Ehdl was significantly up-regulated in both overexpressing lines at 1.5 and 2 fold (Fig.5C). However, MADS51 expression was largely suppressed in the overexpressing lines (Fig.5D). 
301 Amongst the FTL genes, $H d 3 a$, and FTL1were found to be up-regulated in both ox 2 and ox 8

302 (Fig.5F,G), whilst the expression of RFT1 slightly decreased (Fig.5E). For the major genes

303 downstream of $H d 3 a$ (Taoka et al. 2011), we found that MADS15 expression was significantly

304 increased (4 to 6 fold) in ox 2 and ox 8 (Fig.5I), and MADS14 was slightly changed in these two

305 lines (Fig.5H). The results from the RNAi lines i2 and i5, showed similar results to those in the

306 WT line (Fig.5). These results suggested that overexpression of OsFTL10 induced early flowering,

307 through direct enhancement of downstream MADS15 expression and feedback regulation of its

308 upstream genes.

310 Overexpression of FTL10 improved drought tolerance in rice

311

312 As OsFTL10 expression can be influenced by different growth regulators and abiotic stresses such

313 as ABA and mannitol treatments, the drought stress tolerance in transgenic plants was investigated.

314 Transgenic and WT seedlings grown under normal conditions with similar vitality were used for

315 treatment (Fig. 6A, D). When water was withheld for 25 days from the seedlings of the OsFTL10

316 overexpressing plants (ox2, ox5, and ox8) and the WT plants, obvious drought stress phenotypes

317 were observed (Fig. 6B). Compared with the WT plants, leaf rolling and browning were delayed

318 in the OsFTL10 overexpressing plants. Three days after re-watering, most of the OsFTL10

319 overexpressing plants survived, whereas the WT plants withered and died (Fig. 6C). The average

320 survival rate for WT, ox 2 , ox 5 and ox 8 plants were $6 \%, 84 \%, 88 \%$ and $91 \%$, respectively (Fig.

321 6G). Similar treatment was performed on OsFTL10 RNAi lines (i1, i2, and i5) and the WT plants,

322 except that water withholding time was reduced to 20 days (Fig.6E). The OsFTL10 RNAi plants 
323 were found to be more sensitive to drought stress, and after re-watering, most of the WT remained

324 viable, whereas the RNAi plants died (Fig. 6F). The survival rate for WT, i1, i2 and i5 plants were

$32592 \%, 14 \%, 6 \%$ and $8 \%$, respectively (Fig. $6 \mathrm{H}$ ). These results suggested correlation of drought

326 tolerance with $O S F T L 10$ expression. In transgenic rice plants overexpressing OsFTL10 acquired

327 significantly drought tolerance, whilst the suppression of OsFTL10 resulted in drought sensitivity.

328

329 Influence of OsFTL10 on the expression of drought stress-related genes

330

331 Environmental stresses and other external signals can induce complex responses in plants

332 including changes in gene transcription, protein synthesis and metabolism. Studies in different

333 plant species have revealed key genes and proteins involved in stress signaling and transcriptional

334 regulation (Ito et al. 2006; Shinozaki and Yamaguchi-Shinozaki 2007; Molina et al. 2008;

335 Nakashima et al. 2011; Rai and Penna 2013; Janiak et al. 2016). To elucidate the mechanisms of

336 OsFTL10 on drought tolerance, the expression of stress-related transcription factors in stable

337 transgenic plants under drought stress was revealed. Seedlings from WT, i5 and ox 8 were collected

338 for drought stress treatment, and RNAs were extracted for qRT-PCR analysis.

$339 O s D R E B 2 A$ is a drought inducible transcription factor found in rice, which shows increased

340 expression upon drought stress treatment and constitutive expression of this gene results in drought

341 tolerance of transgenic plants (Ito et al. 2006). The expression of this gene was rapidly induced in

342 WT plants and ox 8 upon drought stress, a response which continued for 24 hours. However, the

343 expression level increased significantly after 8 hours of treatment in ox 8 compared to WT plants.

344 In i5, the expression of $O s D R E B 2 A$ decreased after 8 hours of treatment (Fig. 6I).

345 bZIP23, a transcription factor that confers ABA-dependent drought resistance in rice (Xiang et al. 
346 2008), has also been shown to be induced by drought stress. Overexpression of this gene resulted

347 in drought tolerance in transgenic plants. The expression of bZIP23 was rapidly induced in WT,

348 ox 8 and i5 plants under drought stress, whilst its expression decreased after 8 hours in both WT

349 and i5 plants. In ox8, the high expression level of this gene was maintained for 24 hours (Fig. 6J).

350 SNACl gene belongs to the stress-related NAC superfamily of transcription factors.

351 SNAClexpression was induced rapidly in WT, i5 and ox8 plants. In ox 8, the expression of this

352 gene showed higher expression levels after induction which were maintained after 24 hours.

353 Together, these results suggested that overexpression of OsFTL10 improved drought stress

354 tolerance in rice by modulating the expression of stress responsive genes (Fig. 6K).

355

356

357 DISCUSSION

358

359 In this report, we studied the function of OsFTL10 and found that overexpression of this gene

360 induced earlier flowering in rice. These data are similar to the well-studied rice florigen Hd3a and

361 RFT1, which promoted rice flowering under short and long day conditions, respectively. We also

362 found that overexpression of OsFTL10 enhanced the tolerance of rice plants to drought stress.

363 In rice, 13 FT like genes have been found based on sequence similarity, but only three genes,

364 OsFTL1, Hd3a and RFT1, have been studied. Amongst these genes, Hd3a and RFT1 have been

365 extensively studied and shown to be responsive for rice flowering under short and long day

366 conditions, respectively (Komiya et al., 2008). Studies have revealed that Hd3a proteins are

367 produced in mature leaves, and then transported to the shoot meristem. Hd3a protein binds 14-3-3

368 in the cytoplasm, and is imported to nucleus to form a complex (FAC), which binds to C box of

369 MADS15 promoter region, to stimulate the expression of MADS15 and initiate flower transition 
370 (Taoka et al. 2011). To determine whether OsFTL10 also formed a FAC complex to stimulate the

371 floral transition, BiFC was performed and showed that OsFTL10 interacted with GF4c in the

372 nucleus. Yeast two-hybrid results also showed that OsFTL10 interacted with major 14-3-3

373 homologs in rice, including GIF14b and GIF14c, two 14-3-3 proteins interacting with Hd3a (Fig.

374 4). We also found that OsFTL10 up-regulated the downstream flowering gene OsMADS15 (Fig.

375 5). These data suggested that OsFTL10 had a function similar to Hd3a.

376 As a homolog of Hd3a, overexpression of OSFTL10 induced early flowering in rice, but its

377 flowering promotion effect was weaker than Hd3a. The number of days to heading in

378 CaMV35S::Hd3a plants was less than 30 days (Supplemental File1) compared to 53 days in

379 CaMV35S::OSFTL10 plants. As overexpression of both genes under the same promoter resulted

380 in a different flowering time, this effect is probably caused by the amino acids composition of the

381 two proteins.. In addition, we found some differences between the two FTLs: 1) OsFTL10 was

382 found to have a high expression level in young leaves (Fig. 1A), whilst Hd3a was normally highly

383 expressed in mature leaves; 2) Although the localization of both OsFTL10 and Hd3a proteins was

384 found in both the cytoplasm and nucleus (Fig. 4), our BiFC results showed the interaction of

385 OsFTL10 and GF14c mainly occurred in the nucleus, whilst Hd3a and GF14b interactions took

386 place in the cytoplasm (Taoka et al. 2011); 3) Overexpression of OsFT10 enhanced the expression

387 of Ehdl (Fig. 5), Ehdl could activate the expression of FTLs, which explains why the expressions

388 of other FTLs were influenced. We also found that the expression of OSMADS51 was suppressed

389 in OsFTL10 transgenic plants (Fig. 5). Thus, the function of OsFTL10 may be slightly different

390 from the major florigens Hd3a and RFT1 in promotion of flowering.

391 Ehdl can promote rice flowering under both SD and LD. Under SD, Ehdl is involved in two

392 modules OsGI-OsCO-Ehdl-Hd3a and OsGI-OsMADS15-Ehdl-Hd3a, in which Ehdlfunctions 
393 upstream of $H d 3 a$ and promotes flowering by directly up-regulating $H d 3 a$ expression (Yan et

394 al.2011). The expression of OSFTL10, together with the increased expression of Ehd1 and $H d 3 a$

395 in overexpression lines may lead to earlier flowering phenotype in the transgenic plants. As Ehdl

396 is a central player which integrates flowering signaling from both SDs and LDs, and is controlled

397 by multiple genes, the feedback regulation of Ehdl by OsFTL10 needs further investigation.

398 OsMADS51 is a flowering promoter under SDs, which is downstream of OsGI (Kim et al.2008).

399 The decreased expression level of this gene in OsFTL10 overexpression lines is somehow in 400 contradictory with their early flowering phenotype. However, the effects of OsMADS51 may be 401 reduced by the increased expression of Ehdl downstream of it. We also found that RFTl, the 402 florigen responsible for flowering under LDs, are slightly down-regulated. We suspect that the 403 expression of this gene was influenced by OSFTL10 or other flowering related genes, as similar 404 effects had been observed between $H d 3 a$ and RFT1. When $H d 3 a$ expression was knocked down by 405 RNAi, RFT1 was activated for promoting flowering under SDs (Komiya et al., 2009).

406 Photoperiod and vernalization are two environmental factors which influence flowering time in 407 many plant species (Khan et al. 2014). In the model plant Arabidopsis, several photoperiod and 408 circadian clock genes have been found to affect the expression of drought stress related genes. 409 Mutations in clock components, such as the triple mutant prr5 (PSEUDO-RESPONSE 410 REGULATOR 5), 7, 9 are more tolerant to high salinity and drought stresses. TOC1 (timing of 411 CAB expression 1) RNAi plants showed improved drought tolerance. GI overexpression plants 412 show enhanced salt sensitivity, whereas gi mutants show enhanced salt tolerance and enhanced 413 survival under drought stress and oxidative stress (Grundy et al., 2015). Phytochrome B (PhyB) is 414 also a negative regulators of dehydration response (Legnaioli et al., 2009). In barley, variation at 415 the photoperiod response and clock genes Ppd-H1 and HvELF3 can affect the expression level of 
416 osmotic stress genes (Habte et al., 2014). Stresses such as drought and salt, can also regulate 417 flowering times (Kazan and Lyons 2016). Early flowering can be induced by drought stress under 418 LD conditions, and ABA can up-regulate the expression of major flower integrators $F T / T S F$ and 419 SOC1 in a photoperiod-dependent manner (Riboni et al. 2013). Interestingly, most of the above 420 flowering related genes were found to be negative regulators of stress responses. OsGI was 421 recently found to be a negative regulator of osmotic stress response in rice (Li et al., 2016). As $422 O s G I$ is a positive regulator of $O S M A D S 51$, mutation of $O s G I$ may lead to reduced expression of 423 OSMADS51. In our OSFTL10 overexpression lines, the expression of OSMADS15 was decreased. 424 This result indicates that overexpression of OsFTL10 might have similar effects with OsGI mutants 425 on drought tolerance. Ghd7 functions in rice as a negative regulator flowering time under long 426 days (Xue et al., 2008), was also found to be a negative regulator of drought tolerance in rice 427 (Weng et al., 2014). As Ghd7 acts as a suppressor of Ehdl under LDs, the increased expression of 428 Ehd1 in OsFTL10 transgenic plants, suggests that drought tolerance conferred by OsFTL10 may 429 be similar to Ghd7 mutant. The link between flowering and drought tolerance was also 430 demonstrated by a recent work. Overexpression of WOX13 under a drought inducible promoter 431 Rab21 resulted in drought resistant and early flowering in rice. In these plants, drought stress and 432 flowering related genes such as $O s D R E B 1 A, O_{S} D R E B 1 F, H d 3 a$ and $M A D S 14$ were up-regulated 433 (Minh-Thu et al., 2018). Although the above analysis provide clues that drought tolerance by 434 OsFTL10 might be related to $O s G I$ and (or) Ghd7, through controlling the expression of MADS51 435 and (or) Ehdl, much work including the analysis of the mutants involved in the flowering signaling 436 pathways are needed to clarify their regulation networks. The OsFTL10 transgenic plants 437 displayed enhanced drought stress tolerance, as revealed by the higher survival rate and better 438 growth performance under stress conditions (Fig. 6). Cis elements related to drought stress and 
439 ABA response were found in the OsFTL10 promoter (Supplemental File2), and qRT-PCR revealed

440 that OsFTL10 expression was induced by both ABA and mannitol treatments (Fig.2). Investigation

441 of the drought related gene expression in the OsFTL10 transgenic lines further confirmed the

442 drought tolerance of the plants (Fig.6). Many transcription factors can be induced by drought stress

443 in rice, and constitutive expression of these transcription factors have been found to improve plant

444 tolerance to drought stress (Shinozaki and Yamaguchi-Shinozaki 2007; Nakashima et al. 2011;

445 Rai and Penna 2013; Janiak et al. 2016). We studied the expression of three transcription factors

446 including OsDREB2A, bZIP23 and SNAC1, and found they were induced to a higher level of

447 expression in the OSFTL10 overexpressing line ox8. These results correlated well with the

448 performance of the drought stress test results. DREBs/CBFs are transcription factors that can be

449 induced by stresses. They can activate the expression of stress-inducible genes by binding to the

450 dehydration-responsive element/C-repeat (DRE/CRT) cis-acting elements in their promoter

451 region. OsDREB2A is a rice DREB homolog which has been found to be

452 induced by salt and drought. The overexpression of $O S D R E B 2 A$ in rice plants showed both

453 drought and salt stress tolerance (Ito et al. 2006). Abscisic acid is regarded as a

454 drought stress hormone in plants. The transcription factor bZIP23 plays an important role in ABA

455 signaling by binding to the promoter of PP2C49 to improve its expression. As a co-receptor, PP2C

456 can increase ABA binding affinity toward the formation of the Receptor-ABA-PP2C complex

457 (Moreno-Alvero et al. 2017). bZIP23 has been also found to improve drought tolerance in rice

458 (Xiang et al., 2008). The SNACl gene belongs to the stress-related NAC superfamily of

459 transcription factors, which has been expressed in plant for improving drought tolerance

460 (Nakashim et al. 2012). Although the induced expression of the above three transcription factors

461 can be considered as evidence of OsFTL10 tolerance to drought stress, mechanistic links between 
462 OsFTL10 and transcription factors were not fully determined.

463

\section{CONCLUSIONS}

465 In summary, results presented in this study reveal that OsFTL10 functioned similarly to Hd3a in 466 rice by promoting floral transition, and overexpression of OsFTL10 improved the drought stress 467 tolerance of the plants. Further investigations on the identification of OSFTL10 regulated target 468 genes will be helpful for fully elucidating the linkage of flowering and drought stress in the 469 monocot model rice.

470

471 ACKNOWLEDGEMENTS

472 We thank Dr. R.A. Jefferson, CAMBIA, Canberra, Australia, for kindly providing the 473 pCAMBIA1301 and pCAMBIA1390 vectors. We would also like to express our gratitude to 474 Rongyu Du for technical assistance.

475

476

477

478

479

Bernal M, Estiarte M, Peñuelas J (2011) Drought advances spring growth phenology of the 480 Mediterranean shrub Erica multiflora. Plant Biol (Stuttg) 13: 252-725.

481 Chardon F, Damerval C (2005) Phylogenomic analysis of the PEBP gene family in cereals. J Mol 482 Evol 61: 579-590.

483 Corbesier L, Vincent C, Jang S, Fornara F, Fan Q, Searle I, Giakountis A, Farrona S, Gissot L, 484 Turnbull C, Coupland G. (2007) FT protein movement contributes to long-distance signaling in 485 floral induction of Arabidopsis. Science 316: 1030-1033. 
486 Deng L, Li L, Zhang S, Shen J, Li S, Hu S, Peng Q, Xiao J, Wu C (2017) Suppressor of rid1

487 (SID1) shares common targets with RID1 on florigen genes to initiate floral transition in rice.

488 PLoS Genet. 13 (2):e1006642. doi:10.1371/journal.pgen.1006642.

489 Franks SJ, Sim S, Weis AE (2007) Rapid evolution of flowering time by an annual plant in 490 response to a climate fluctuation. Proc Natl Acad Sci USA 104: 1278-1282.

491 Franks, SJ (2011) Plasticity and evolution in drought avoidance and escape in the annual plant 492 Brassica rapa. New Phytol 190: 249-257.

493 Grundy J, Stoker C, Carre IA (2015) Circadian regulation of abiotic stress tolerance in plants.

494 Frontiers in plant science 6, 648.

495 Habte E, Muller LM, Shtaya M, Davis SJ, von Korff M (2014) Osmotic stress at the barley root 496 affects expression of circadian clock genes in the shoot. Plant Cell Environ 37, 1321-1327.

497 Ito Y, Katsura K, Maruyama K, Taji T, Kobayashi M, Seki M, Shinozaki K, Yamaguchi-Shinozaki 498 K (2006) Functional analysis of rice DREB1/CBF-type transcription factors involved in cold499 responsive gene expression in transgenic rice. Plant Cell Physiol 47: 141-153.

500 Iuchi S, Kobayashi M, Taji T, Naramoto M, Seki M, Kato T, Tabata S, Kakubari Y, Yamaguchi501 Shinozaki K, Shinozaki K (2001) Regulation of drought tolerance by gene manipulation of 9-cis502 epoxycarotenoid dioxygenase, a key enzyme in abscisic acid biosynthesis in Arabidopsis. Plant J $503 \quad 27: 325-333$.

504 Izawa T, Oikawa T, Sugiyama N, Tanisaka T, Yano M, Shimamoto K (2002) Phytochrome 505 mediates the external light signal to repress FT orthologs in photoperiodic flowering of rice. Genes 506 Dev 16: 2006-2020.

507 Janiak A, Kwaśniewski M, Szarejko I (2016) Gene expression regulation in roots under drought. 508 J Exp Bot 67: 1003-1014. 
509 Jefferson RA (1987) Assaying chimeric genes in plants: The Gus gene fusion system. Plant Mol

510 Biol Rep 5: 387-405.

511 Kardailsky I, Shukla VK, Ahn JH, Dagenais N, Christensen SK, Nguyen JT, Chory J, Harrison

512 MJ, Weigel D (1999) Activation tagging of the floral inducer FT. Science 286: 1962-1965.

513 Kazan K, Lyons R (2016) The link between flowering time and stress tolerance. J Exp Bot 67: 47-

51460.

515 Khan MR, Ai XY, Zhang JZ (2014) Genetic regulation of flowering time in annual and perennial 516 plants. Wiley Interdiscip Rev RNA 5: 347-359.

517 Kim SK, Yun CH, Lee JH, Jang YH, Park HY, Kim JK (2008) OsCO3, a CONSTANS-LIKE

518 gene, controls flowering by negatively regulating the expression of FT-like genes under SD

519 conditions in rice. Planta 228, 355-365.

520 Kobayashi Y, Kaya H, Goto K, Iwabuchi M, Araki T (1999) A pair of related genes with

521 antagonistic roles in mediating flowering signals. Science 286: 1960-1962.

522 Kobayashi K, Yasuno N, Sato Y, Yoda M, Yamazaki R, Kimizu M, Yoshida H, Nagamura Y,

523 Kyozuka J (2012). Inflorescence meristem identity in rice is specified by overlapping functions

524 of three AP1/FUL-like MADS box genes and PAP2, a SEPALLATA MADS box gene. Plant

525 Cell. 24(5):1848-59.

526 Kojima S, Takahashi Y, Kobayashi Y, Monna L, Sasaki T, Araki T, Yano M (2002) Hd3a, a rice

527 ortholog of the Arabidopsis FT gene, promotes transition to flowering downstream of Hd1 under

528 short-day conditions. Plant Cell Physiol 43: 1096-1105.

529 Komiya R, Ikegami A, Tamaki S, Yokoi S, Shimamoto K (2008) Hd3a and RFT1 are essential for

530 flowering in rice. Development 135: 767-774.

531 Komiya R, Yokoi S, Shimamoto K (2009) A gene network for long-day flowering activates RFT1 
532 encoding a mobile flowering signal in rice. Development 136: 3443-3450.

533 Kooyers NJ (2015) The evolution of drought escape and avoidance in natural herbaceous 534 populations. Plant Sci 234: 155-162.

535 Legnaioli T, Cuevas J, Mas P (2009) TOC1 functions as a molecular switch connecting the 536 circadian clock with plant responses to drought. EMBO J 28, 3745-3757.

537 Li MR, Li HQ (2003) A simple and highly efficient Agrobacterium-mediated rice transformation 538 system. Acta Biol Exp Sin 36: 289-294.

539 Li M, Lin X, Li H, Pan X, Wu G (2011) Overexpression of AtNHX5 improves tolerance to both 540 salt and water stress in rice (Oryza sativa L.). Plant Cell Tiss Organ Cult 107(2): 283-293.

541 Li M, Li H, Hu X, Pan X, Wu G (2011) Genetic transformation and overexpression of a rice Hd3a 542 induces early flowering in Saussurea involucrata Kar. et Kir. ex Maxim. Plant Cell Tiss Organ 543 Cult 106:363-371.Lin MK, Belanger H, Lee YJ, Varkonyi-Gasic E, Taoka K, Miura E, 544 Xoconostle-Cázares B, Gendler K, Jorgensen RA, Phinney B, Lough TJ, Lucas WJ (2007) 545 FLOWERING LOCUS T protein may act as the long distance florigenic signal in the cucurbits. 546 Plant Cell 19: 1488-1506.

547 Lin Q, Zhou Z, Luo W, Fang M, Li M, Li H (2017) Screening of proximal and interacting proteins 548 in rice protoplasts by proximity-dependent biotinylation. Front Plant Sci 8:1-10.

549 Matsubara K, Yamanouchi U, Nonoue Y, Sugimoto K, Wang ZX, Minobe Y, Yano M (2011)

550 Ehd3, encoding a plant homeodomain finger-containing protein, is a critical promoter of rice 551 flowering. Plant J 66, 603-612.

552 Molina C, Rotter B, Horres R, Udupa SM, Besser B, Bellarmino L, Baum M, Matsumura H, 553 Terauchi R, Kahl G, Winter P (2008) SuperSAGE: the drought stress-responsive transcriptome of 
554 chickpea roots. BMC Genomics 9: 553.

555 Moreno-Alvero M, Yunta C, Gonzalez-Guzman M, Lozano-Juste J, Benavente JL, Arbona V,

556 Menéndez M, Martinez-Ripoll M, Infantes L, Gomez-Cadenas A, Rodriguez PL, Albert A (2017)

557 Structure of ligand-bound intermediates of crop ABA receptors highlights PP2C as necessary ABA 558 co-receptor. Mol Plant 10: 1250-1253.

559 Nakashima K, Takasaki H, Mizoi J, Shinozaki K, Yamaguchi-Shinozaki K (2011) NAC 560 transcription factors in plant abiotic stress responses. Biochim Biophys Acta 1819: 97-103.

561 Qin X, Zeevaart JA (1999) The 9-cis-epoxycarotenoid cleavage reaction is the key regulatory step 562 of abscisic acid biosynthesis in water-stressed bean. Pro Natl Acad Sci USA 96: 15354-1561.

563 Rai AN, Penna S (2013) Molecular evolution of plant P5CS gene involved in proline biosynthesis.

564 Mol Biol Rep 40: 6429-6435.

565 Riboni M, Galbiati M, Tonelli C, Conti L (2013) GIGANTEA enables drought escape response 566 via abscisic acid-dependent activation of the florigens and SUPPRESSOR OF 567 OVEREXPRESSION OF CONSTANS. Plant Physiol 162: 1706-1719.

568 Sherrard ME, Maherali H (2006).The adaptive significance of drought escape in Avena barbata, 569 an annual grass. Evolution 60: 2478-2489.

570 Shinozaki K, Yamaguchi-Shinozaki K (2007) Gene networks involved in drought stress response 571 and tolerance. J Exp Bot 58: 221-227.

572 Song YH, Shim JS, Kinmonth-Schultz HA, Imaizumi T (2015) Photoperiodic flowering: time 573 measurement mechanisms in leaves. Annu Rev Plant Biol 66: 441-464.

574 Tamaki S, Matsuo S, Wong HL, Yokoi S, Shimamoto K (2007) Hd3a protein is a mobile flowering 575 signal in rice. Science 316: 1033-1036.

576 Taoka K, Ohki I, Tsuji H, Furuita K, Hayashi K, Yanase T, Yamaguchi M, Nakashima C, Purwestri 
577 YA, Tamaki S, Ogaki Y, Shimada C, Nakagawa A, Kojima, C, Shimamoto K (2011) 14-3-3

578 proteins act as intracellular receptors for rice Hd3a florigen. Nature 476: 332-335.

579 Taylor IB, Burbidge A, Thompson AJ (2000) Control of abscisic acid synthesis. J Exp Bot 51:

$580 \quad 1563-1574$.

581 Tsuji H (2017) Molecular function of florigen. Breed Sci 67: 327-332.

582 Tsuji H, Taoka K, Shimamoto K (2011) Regulation of flowering in rice: two florigen genes, a 583 complex gene network, and natural variation. Curr Opin Plant Biol 14: 45-52.

584 Turck F, Fornara F, Coupland G (2008) Regulation and identity of florigen: FLOWERING 585 LOCUS T moves center stage. Annu Rev Plant Biol 59: 573-594.

586 Wagner D (2017) Key developmental transitions during flower morphogenesis and their 587 regulation. Cur Opin Genet Dev 45: 44-50.

588 Wan L, Zhang J, Zhang H, Zhang Z, Quan R, Zhou S, Huang R (2011) Transcriptional activation 589 of OsDERF1 in OsERF3 and OsAP2-39 negatively modulates ethylene synthesis and drought 590 tolerance in rice. PLoS ONE 6: e25216

591 Wang Q, Guan Y, Wu Y, Chen H, Chen F, Chu C (2008) Overexpression of a rice OsDREB1F 592 gene increases salt, drought, and low temperature tolerance in both Arabidopsis and rice. Plant 593 Mol Biol 67: 589-602.

594 Wang Z, Chen C, Xu Y, Jiang R, Han Y, Xu Z, Chong K (2004) A practical vector for efficient 595 knockdown of gene expression in rice. Plant Mol Bio Rep 22: 409-417.

596 Weng X, Wang L, Wang J, Hu Y, Du H, Xu C, Xing Y, Li X, Xiao J, Zhang Q (2014) Grain 597 number, plant height, and heading date7 is a central regulator of growth, development, and stress 598 response. Plant Physiol 164: 735-747.

599 Wigge PA, Kim MC, Jaeger KE, Busch W, Schmid M, Lohmann JU, Weigel D (2006) Integration 
600 of spatial and temporal information during floral induction in Arabidopsis. Science 309: 10566011059.

602 Xiang Y, Tang N, Du H, Ye H, Xiong L (2008) Characterization of OsbZIP23 as a key player of 603 the basic leucine zipper transcription factor family for conferring abscisic acid sensitivity and 604 salinity and drought tolerance in rice. Plant Physiol 148: 1938-1952.

605 Xue W, Xing Y, Weng X, Zhao Y, Tang W, Wang L, Zhou H, Yu S, Xu C, Li X, Zhang, Q (2008) 606 Natural variation in Ghd7 is an important regulator of heading date and yield potential in rice. Nat 607 Genet 40: 761-767.

608 Yan WH, Wang P, Chen HX, Zhou HJ, Li QP, Wang CR, Ding ZH, Zhang YS, Yu SB, Xing YZ, 609 Zhang QF (2011) A major QTL, Ghd8, plays pleiotropic roles in regulating grain productivity, 610 plant height, and heading date in rice. Molecular Plant 4, 319-330.

611 Yin X, Rocha PSCF, Wang M, Zhu Y, Li L, Song S, Xia X (2011) Rice gene OsDSR-1 promotes 612 lateral root development in Arabidopsis under high-potassium conditions. J Plant Bio 54: 180613189. 


\section{Figure 1}

Temporal and spatial expression OsFTL10 in rice

(A) The expression of OsFTL10 in rice tissues by qRT-PCR analysis.(B) GUS assay of OsFTL10 promoter activity in immature leaf. (C) Mature leaf. (D) Flower. $(E, F)$ Young panicle. $(G)$ Anther. (H) Germinated seedling (with amplified leaf tip). 


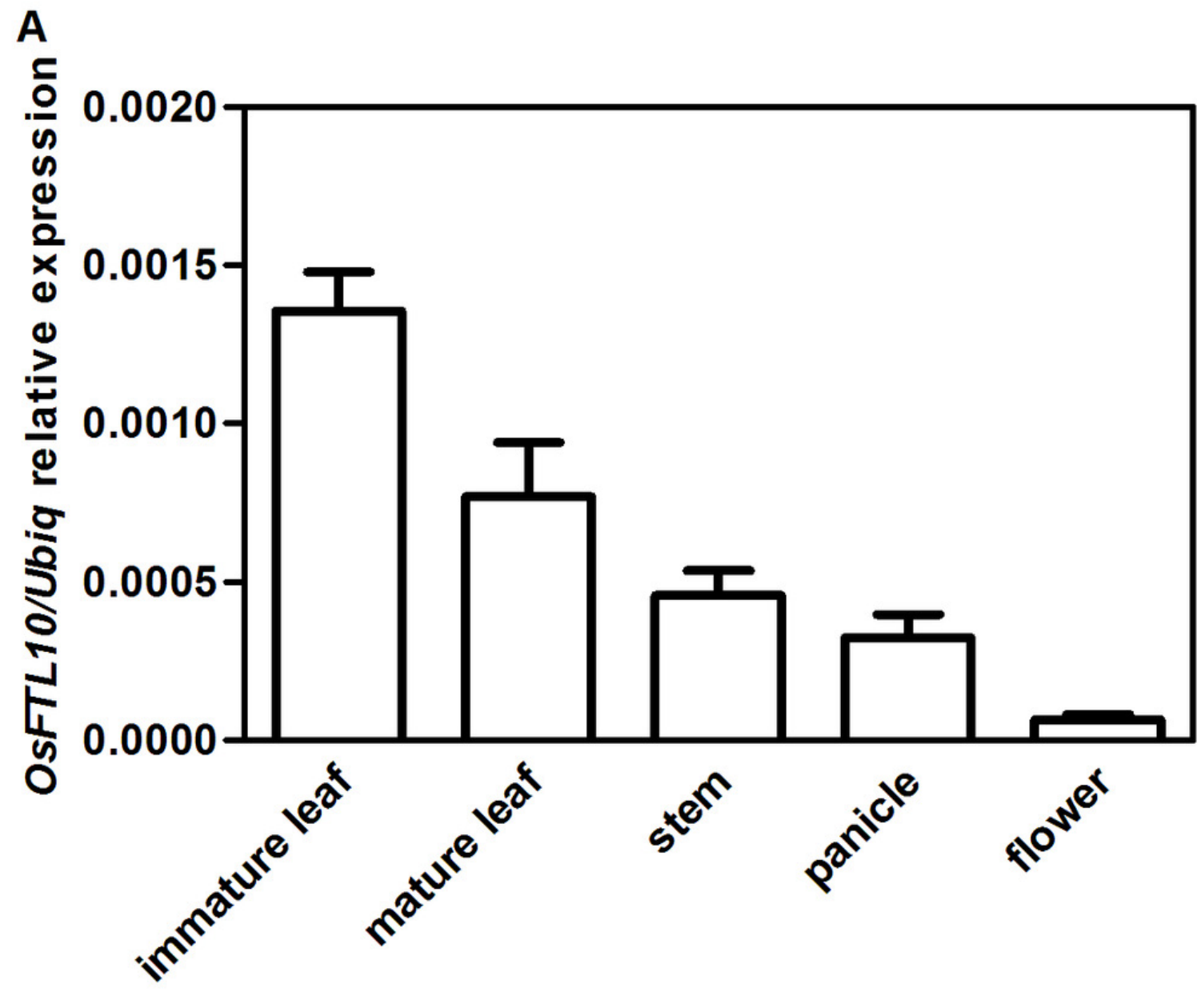

B

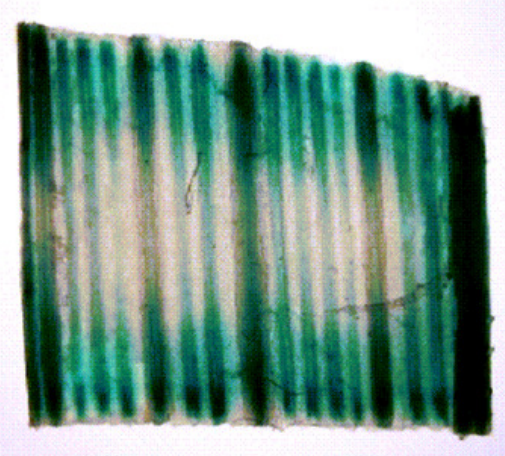

E

F

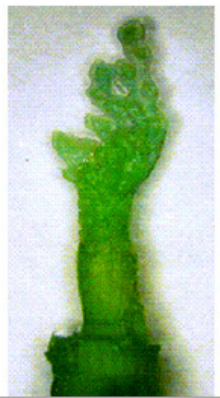

C

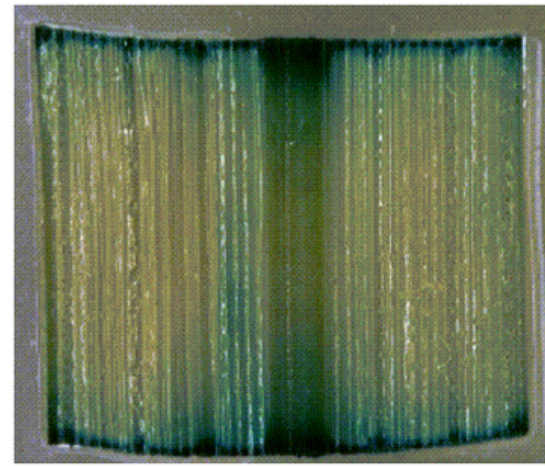

G

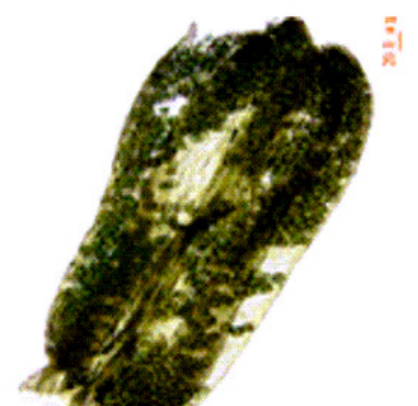

D

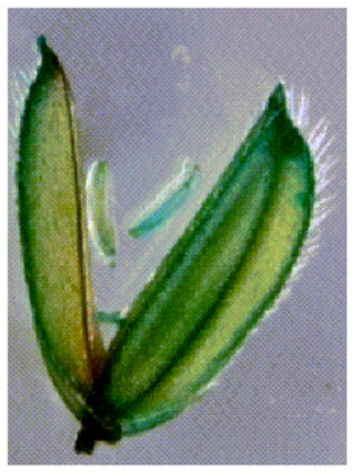

H

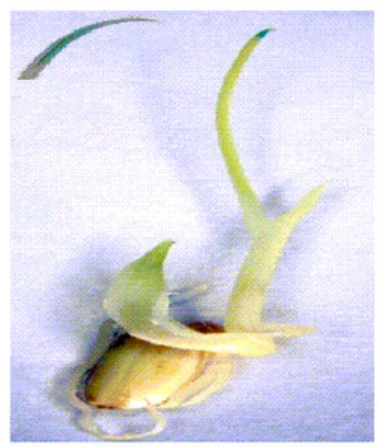


Figure 2

Expression of OsFTL10 in rice seedlings treated with different growth regulators and stresses

Expression of OSFTL10 in rice seedlings treated with IAA (A). (B) BA. (C) ABA. (D) SA. (E) MeJA. (F) GA. (G) Mannitol. (H) NaCl. All data represent the mean of three biological replicates, with error bars indicating SD. $(*$ indicates a significant difference in comparison with the control (Oh) at $P<0.05)$. 

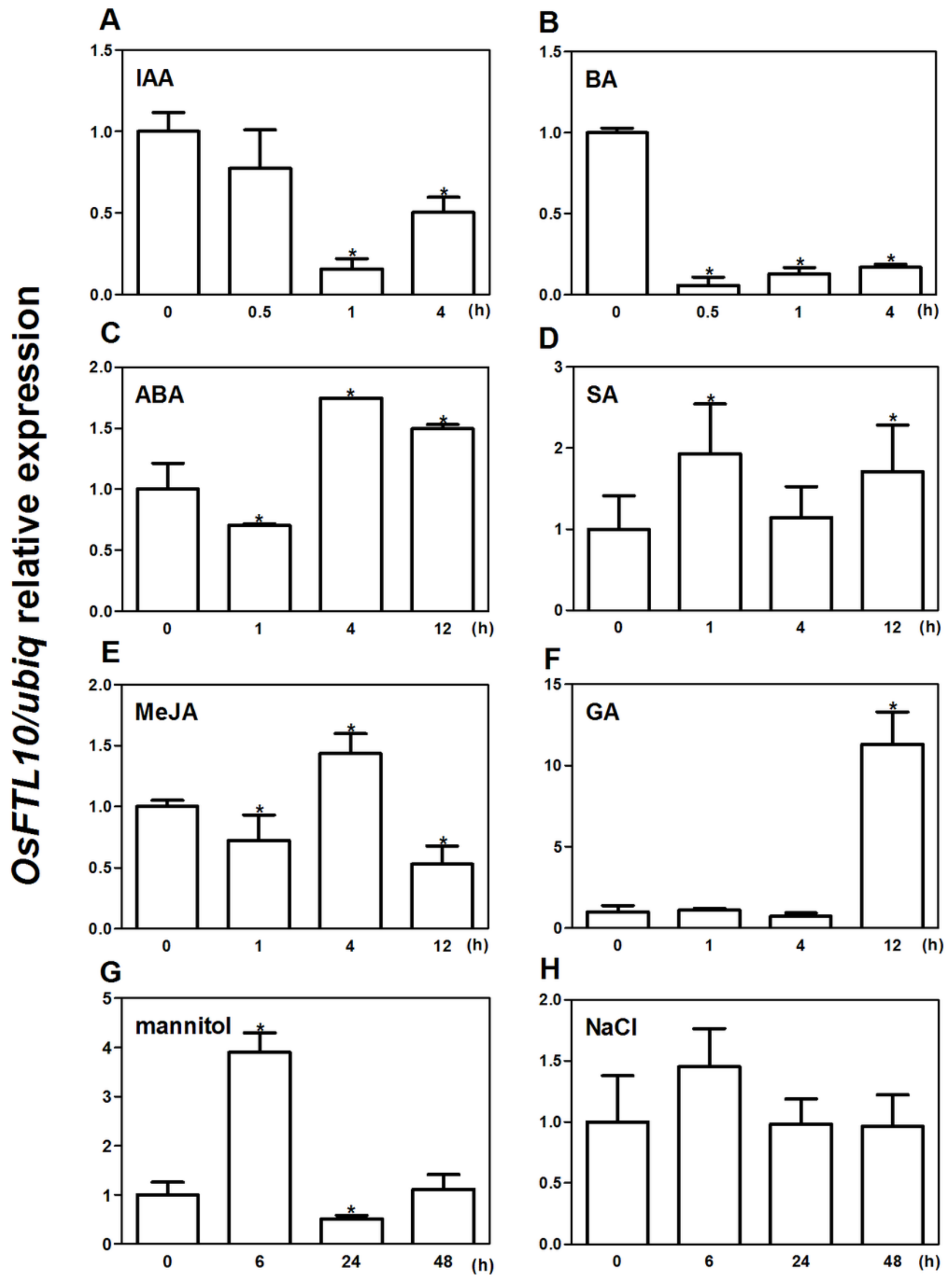


\section{Figure 3}

Phenotype of FTL10 overexpression and RNAi transgenic plants

(A) Representative picture of WT and overexpression line ox8 after heading .(B)

Representative picture of WT and RNAi line i5 after heading. Performance of the transgenic lines greenhouse on heading date (C). Grain weight (D). Number of flowers (E). Plant height $(F)$. Data represent the mean of 15 to 24 plants, with error bars indicating SD. ${ }^{*}$ indicates a significant difference in comparison to the $W T$ at $P<0.05)$. 
A
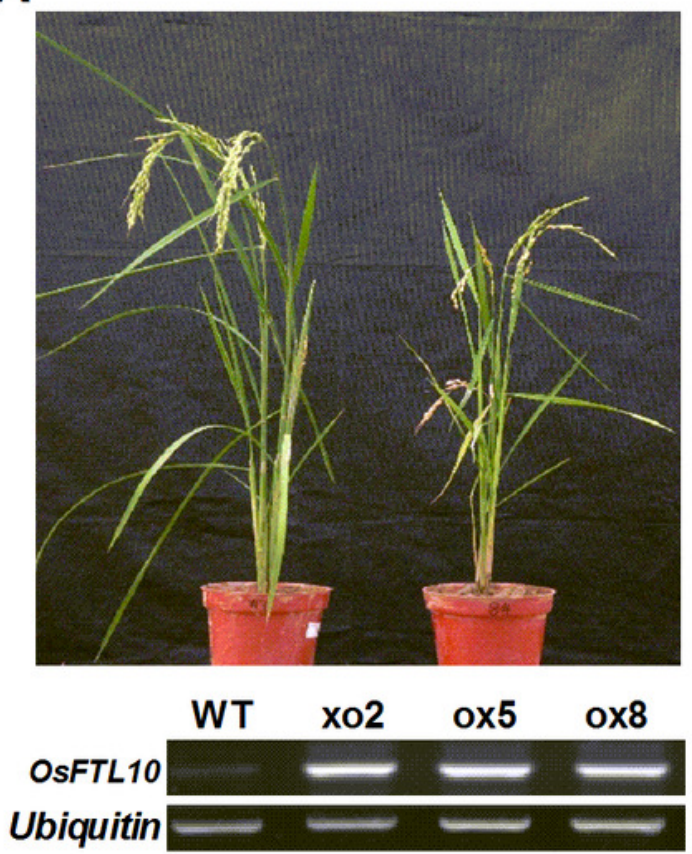

C

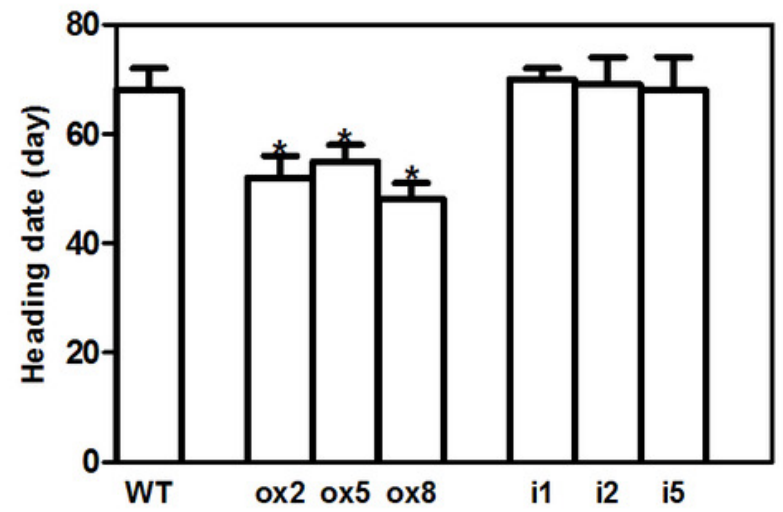

E

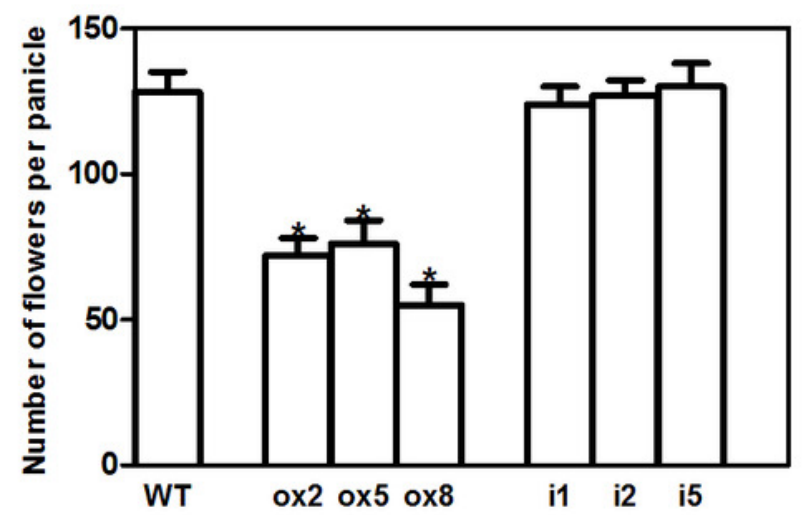

B
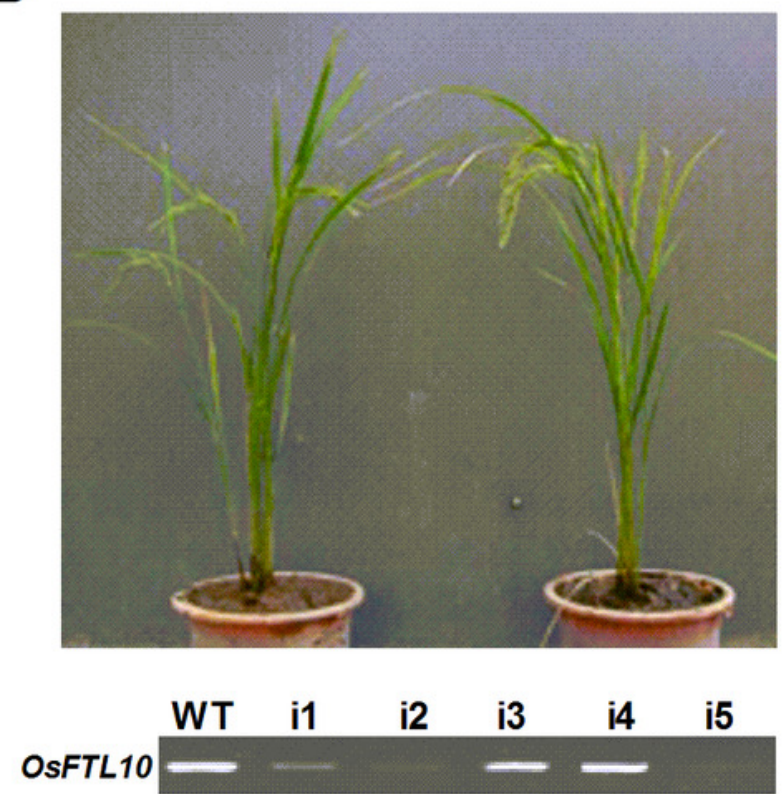

Ubiquitin

D

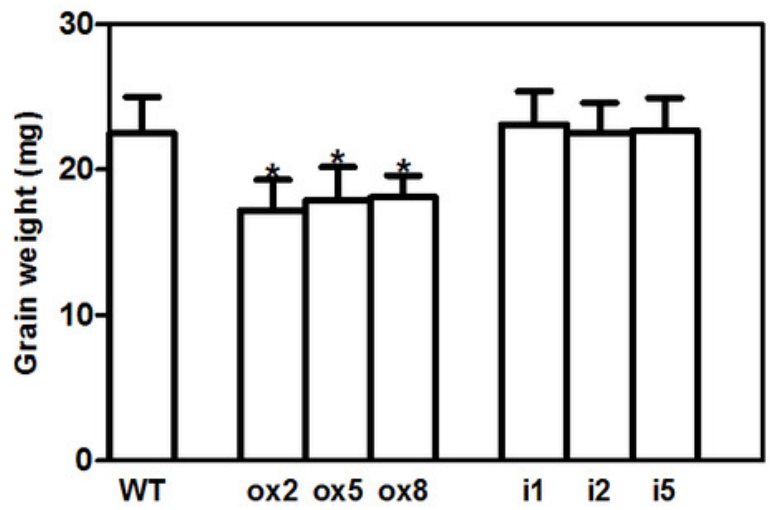

$\mathbf{F}$

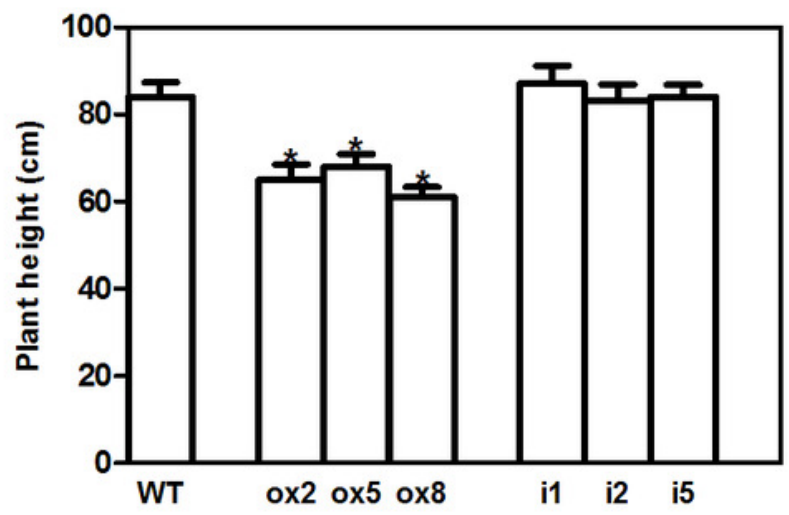


Figure 4

BiFC and $\mathrm{Y} 2 \mathrm{H}$ analysis of OsFTL10 interaction with $14-3-3 \mathrm{C}$

(A) Subcellular localization of OsFTL10, 14-3-3c. (B) BiFC analysis of OsFTL10 interaction with

14-3-3c in rice protoplasts. (C) Y2H analysis of interaction of OsFTL10 with different 14-3-3s.
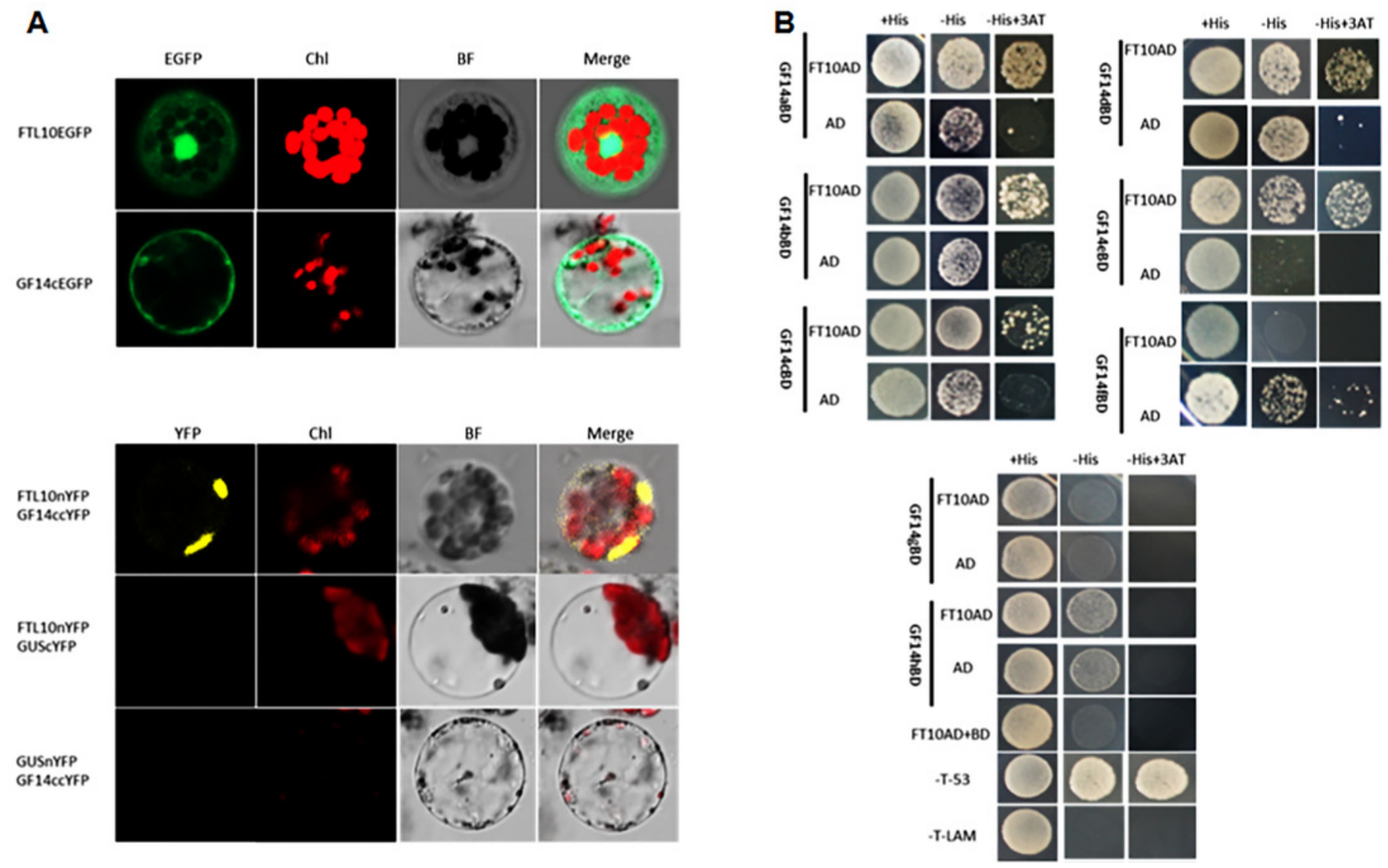
Figure 5

Expression of major flowering related genes in transgenic plants

Expression of OsGl (A), Hd1 (B), Ehd1 (C), MADS51 (D), RFT1 (E) , Hd3a (F) , FTL1 (G)

,MADS14 (H),MADS15 (I) in transgenic plants. All data represent the mean of three biological replicates, with error bars indicating SD. ${ }^{*}$ indicates a significant difference in comparison to the WT at $P<0.05)$.

A

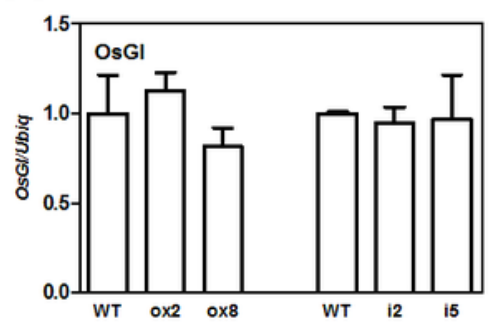

D

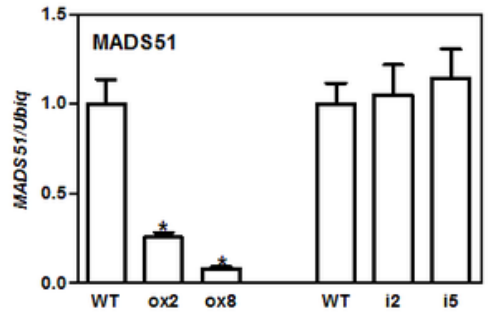

G

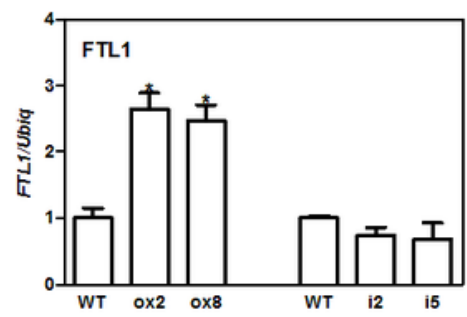

B

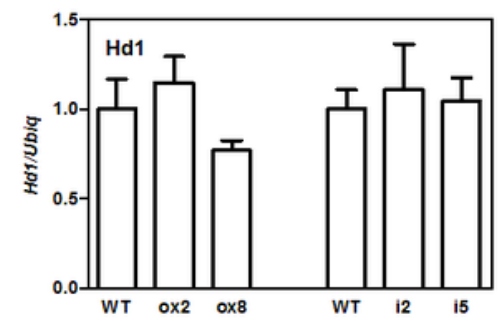

E

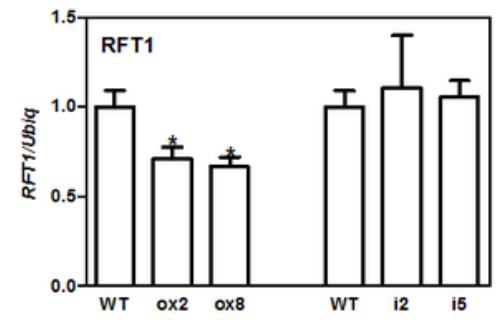

H

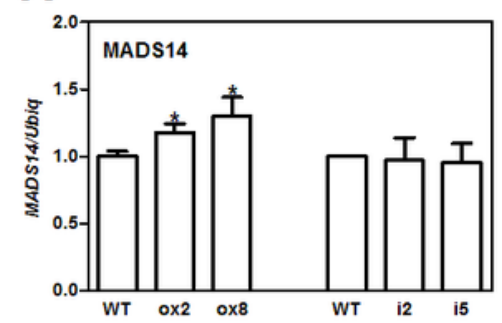

C

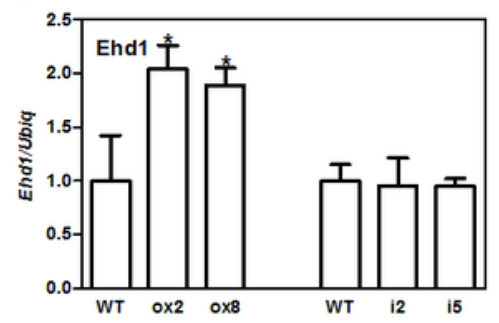

$\mathbf{F}$

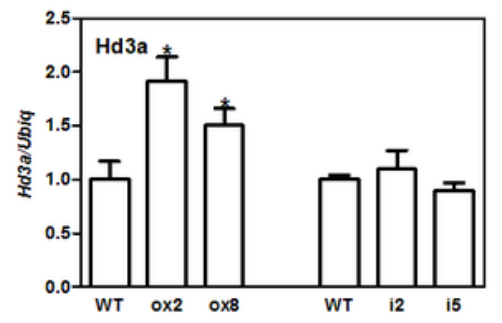

I

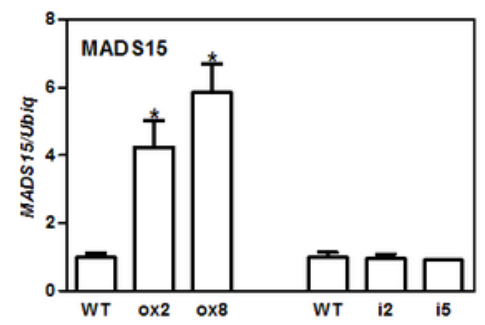




\section{Figure 6}

Representative picture of drought tolerance test of transgenic plants and expression of drought stress related genes in transgenic plants

Phenotypic comparison of the transgenic rice plants before $(A, D)$, and after water stress ( $B$, $E)$, and after recovery for 3 days $(C, F) .(G, H)$ Survival rates of transgenic plants after drought stress treatment. Data represent the mean of three replicates, with error bars indicating SD. (* indicates a significant difference in comparison to the WT at $\mathrm{P}<0.05$ ). Expression of stress related genes OSDREB2A (I), bZIP23(J), and SNAC1 (K) in WT and transgenic plants after drought treatment for different time. Data represent the mean of three replicates, with error bars indicating SD. $(*$ indicates a significant difference in comparison to the WT with the same treatment time at $P<0.05$ ). 


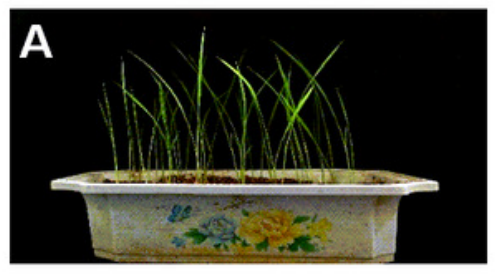

WT ox2 ox5 ox8

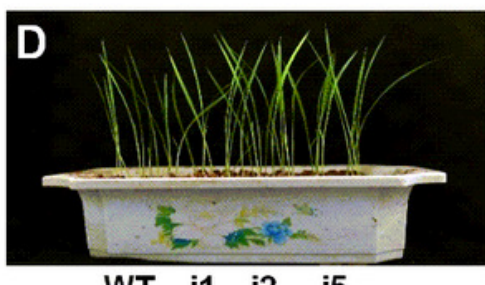

WT i1 i2 i5
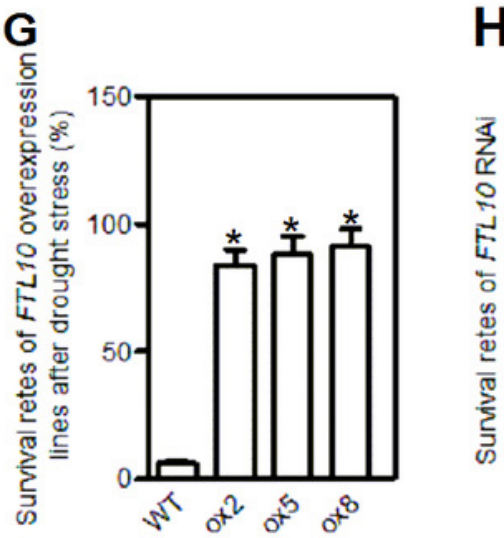

$J$

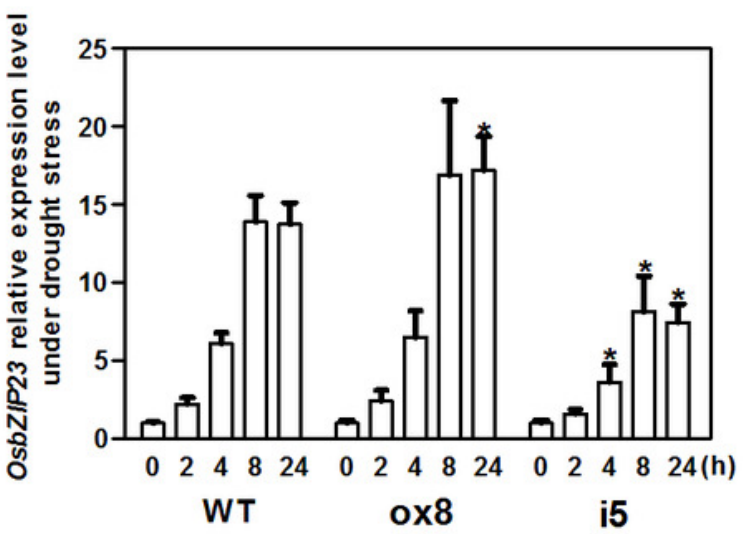

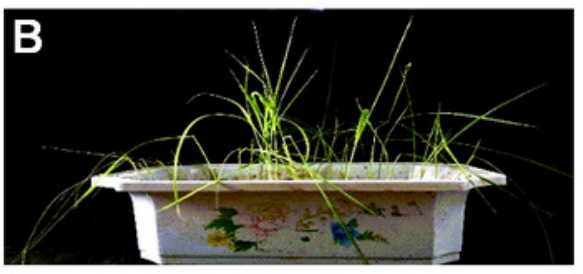

WT ox2 ox5 ox8

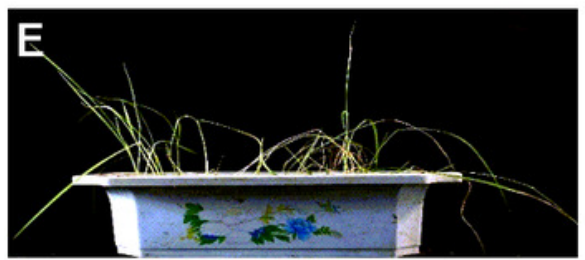

WT i1 i2 i5

$\mathbf{H}$

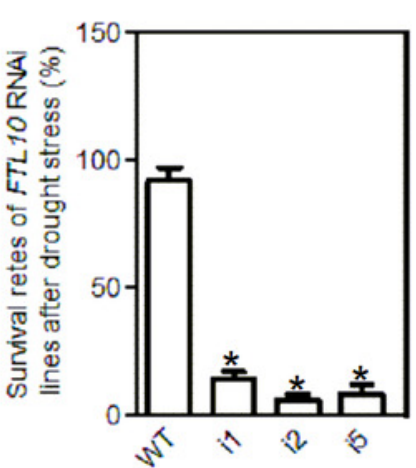

i5

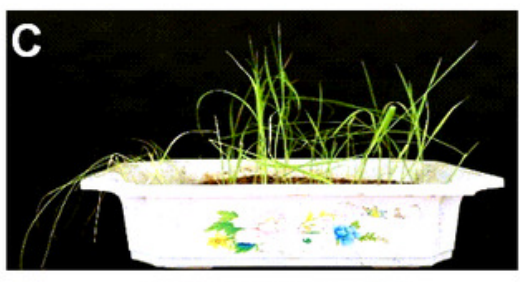

WT ox2 ox5 ox8

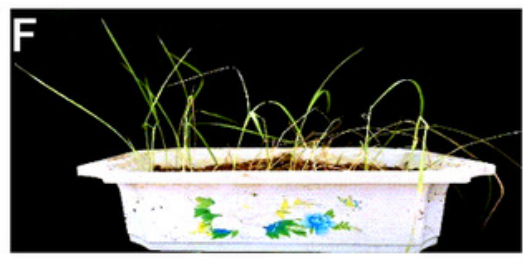

WT i1 i2 i5
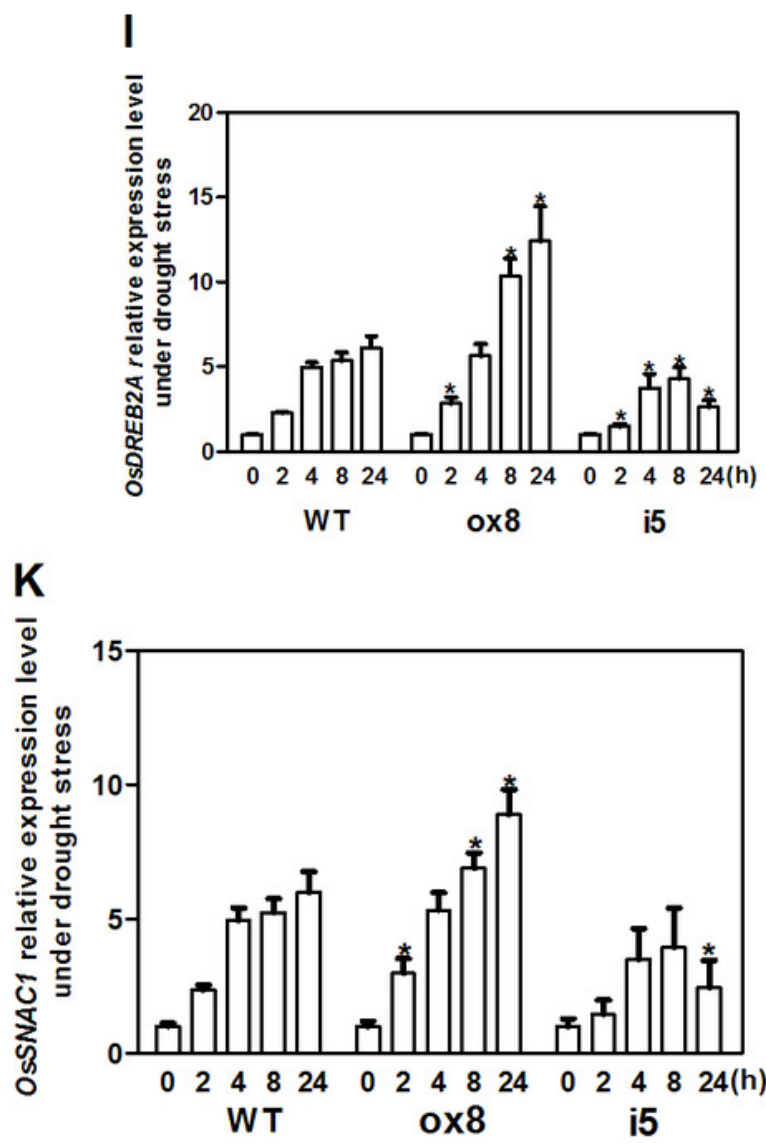